\title{
UEAtc
}

UNIÓN EUROPEA PARA LA IDONEIDAD TÉCNICA EN LA CONSTRUCCIÓN noviembre, 1995

\section{INFORME TÉCNICO DE LA UEAtc PARA LA SELECCIÓN Y EMPLEO DE LOS TERMOPLÁSTICOS RECICLADOS EN LOS PRODUCTOS DE CONSTRUCCIÓN CON EVALUACIÓN}

800-3 
El presente Documento ha sido elaborado por:

-El British Board of Agrement (BBA), representando al Reino Unido,

-El Stichting Bouwkwaliteit (SBK), representando a los Países Bajos.

-Le Centre Scientifique et Technique du Bâtiment (CSTB), representando a Francia,

-El Statens Byggeforskningsinstitut (SBI), representando a Dinamarca,

-El Forschungsgesellschaft für Wohnen, Bauen und Planen (FGW) (Viena), representando a Austria,

-El Instituto de Ciencias de la Construción Eduardo Torroja (IETCC), representando a España,

-El Instituto Centrale per l'Industrializzazione et la Tecnologia Edilizia (ICITE), representando a Italia,

-El Laboratorio Nacional de Engenharia Civil (LNEC), representando a Portugal,

-La Union Belge pour l'Agrément technique dans la construction (UBAtc), representando a Bélgica,

-El Technical Research Center of Finland (VTT), representando a Finlandia

-El Norwegian Building Research Institute (NBI), representando a Noruega,

-El Irish Agrement Board (IAB), representando a Irlanda,

-El Epitesügyi Minöségellenörzö Intézet (EMI), representanđo a Hungría

-El Instytut Techniki Budowlanej (ITB), representado a Polonia,

-El Swedish Institute for Technical Approval (SITAC), representado a Suecia,

El British Board of Agrement actuó como ponente.

Ha participado en los trabajos y contribuido a su elaboración, una delegación de la Asociación de fabricantes de Plásticos en Europa (APME).

TRADUCCIÓN Y ADAPTACIÓN: J.M. Bielza, Dr. en C. Químicas.

2 


\section{ÍNDICE}

1. INTRODUCCIÓN

2. OBJETO 4

3. TERMINOLOGÍA - DEFINICIONES

4. PROBLEMÁTICA DE LA EVALUACIÓN 5

4.1. General 5

4.2. Factores a considerar 5

4.3. Procedimientos de evaluación 6

4.4. Evaluación de la durabilidad 6

5. EVALUACIÓN BASADA EN LA PROCEDENCIA DE LOS TERMOPLÁSTICOS RECICLADOS 7

$\begin{array}{ll}\text { 5.1. Termoplásticos reutilizados } & 7\end{array}$

5.2. Termoplásticos reprocesados

5.3. Termoplásticos reciclados después de su uso (origen industrial)

5.4. Termoplásticos reciclados después de su uso (origen doméstico) 8

\section{ANEXOS}

A) Efecto de los agentes de envejecimiento sobre los termoplásticos 8

B) Características para la identificación o evaluación 9

C) Recomendaciones relativas al nivel de control de calidad y a la importancia $\begin{array}{ll}\text { de la acción de los agentes ambientales } & 10\end{array}$

D) Ejemplos de productos de construcción susceptibles de incluir termoplásticos reciclados. $\quad 11$ 


\section{INTRODUCCIÓN}

El reciclado (recuperación y reutilización) de termoplásticos es un campo de actividad de interés e importancia creciente. La previsiones de orden económico, financiero y legislativo, deberían afirmar esta tendencia en los próximos años.

Algunos miembros de la UEAtc (BBA, CSTB) han recibido solicitudes de evaluación correspondientes a productos en los que se incluyen termoplásticos reciclados. El contenido en termoplásticos reciclados de los productos presentados para evaluación puede variar desde un porcentaje bajo, hasta el $100 \%$. La inclusión de termoplásticos reciclados puede examinarse bien en el momento de la evaluación inicial o como modificación posterior de la especificación técnica de un producto ya evaluado.

El presente documento se ha elaborado con el fin de identificar los problemas relacionados con el uso de termoplásticos reciclados y establecer un acuerdo para la evaluación de estos productos. Se dedica igualmente a normalizar la terminología y definiciones utilizadas, pues este aspecto supone un importante origen de confusiones y malentendidos. El objeto del informe no es el de presentarun estudio en profundidad de los procesos de degradación susceptibles de afectar a los termoplásticos ni de definir todos los casos que permitan el uso de termoplásticos reciclados.

Los termoplásticos pueden reciclarse de varias formas. Pueden recuperarse y después transformarse en productos que presenten características análogas a las del original (reciclado primario) o productos que presentan características sensiblemente diferentes (reciclado secundario). Son los tipos de reciclado que presentan más interés para los miembros de la UEAtc. Así, la reducción de termoplásticos en productos químicos elementales o combustibles (reciclado terciario), o la recuperación del contenido energético de estos materiales para su uso como combustibles no son campos en los que vaya a intervenir la UEAtc.

\section{OBJETO}

El presente Informe Técnico sólo trata del uso de termoplásticos objeto de un reciclado primario o secundario, utilizados en productos de construcción presentados a evaluación.

En la actualidad se reciclan varios tipos de termoplásticos; pero los más utilizados en la fabricación de productos de construcción son el polietileno (PE) de alta (HD) y baja densidad (LD), el polipropileno (PP) y el policloruro de vinilo (PVC) rígido y plastificado, y son a los que se refie- re este informe. Sin embargo, puede utilizarse el mismo planteamiento para otros termoplásticos.

Estos termoplásticos se utilizan, principalmente, para la fabricación de perfiles, canalizaciones, placas y películas $y$ son a las que se refiere este informe. El anexo D recoge ejemplos de tipos de productos en los que pueden utilizarse termoplásticos reciclados para su fabricación.'

\section{TERMINOLOGIA - DEFINICIONES}

Los términos y definiciones señalados con el símbolo $\left(^{*}\right)$ se han extraído del documento ISO/CD 472/DAM 6 Plásticos - Vocabulario - $6^{\circ}$ proyecto: "Términos relativos al reciclado". Algunas de las definiciones están en estudio y pueden sufrir algún cambio. Para este documento se han seleccionado los siguientes términos y definiciones:

\section{Degradación}

Destrucción o alteración de las propiedades físicas y/o químicas de un termoplástico bajo la acción de agentes de degradación, solos o combinados ${ }^{1}$.

\section{Reciclado de los termoplásticos*}

Proceso por el que los termoplásticos que han alcanzado el estado de residuo sólido se agrupan, separan y transforman, para volver a utilizarse:

- reciclado primario*

transformación de residuos termoplásticos en un producto cuyas características son análogas a las del producto original;

\section{- reciclado secundario*}

transformación de residuos termoplásticos en un producto diferente al producto original, pero que presentan características análogas ${ }^{2}$;

\section{- reciclado terciario*}

producción de productos químicos de base o combustibles a partir de termoplásticos separados o residuos que forman parte de los residuos urbanos;

\section{- reciclado cuaternario*}

recuperación del contenido energético de los residuos termoplásticos para su utilización como combustible.

\footnotetext{
1) Si la degradación se produce por la acción de las condiciones climáticas, se puede utilizar el término de envejecimiento ambiental.

2) En caso de duda, el Organismo de evaluación debe decidir si el material ha sido producido por reciclado primario o secundario.

3) No tratado en este documento.
} 


\section{Termoplásticos reciclados}

Termoplásticos, de cualquier origen que han sido transformados y recuperados para su posterior utilización.

\section{Termoplásticos reciclados después de su utilización*}

Termoplásticos reciclados, recuperados de productos que han cumplido su función primaria. Pueden provenir de origen industrial o doméstico (p.ej. puntos de recogida de basuras, etc.).

\section{Termoplásticos reciclados antes de su utilización*}

Termoplásticos reciclados, recuperados para reutilización a partir de productos o materiales que no han cumplido su función primaria. Puede tratarse de termoplásticos reutilizados* (p.ej. colas, orillos, rebabas, desechos de termoplásticos que no han sufrido un reciclado primario, de la propia cadena de producción); o termoplásticos reprocesados* (p.ej. elaborados a partir de residuos industriales de un tercero).

\section{Termoplásticos puros}

Termoplásticos en forma de pastilla, granza, polvo, filamento o liquido, que no han sufrido otra utilización o transformación que la necesaria para su fabricación.

\section{PROBlemas ASOCIADOS CON LA EVALUACIÓN}

\subsection{General}

El objeto de una evaluación es determinar la aptitud al uso previsto del producto en cuestión y estimar el período durante el cual el producto conservará las propiedades que le permitan cumplir eficazmente su función, teniendo en cuenta las condiciones de utilización.

La evaluación de la durabilidad generalmente se basa en los resultados de los ensayos acelerados realizados en condiciones precisas. Después se interpretan estos resultados para avanzar una estimación de vida mínima esperada. Se requiere que la interpretación tenga un grado de confianza razonable. Como en todos los materiales, es esencial que las características de los termoplásticos reciclados utilizados no varien de tal forma que sea imposible relacionar los resultados de los ensayos de un limitado número de muestras sobre la producción total.

\subsection{Factores a considerar}

Los factores que afectan a la idoneidad de los productos, conteniendo termoplásticos reciclados son los siguientes: a) origen de los termoplásticos reciclados y los controles efectuados para su selección y utilización

En los termoplásticos puros, las variaciones en las propiedades son pequeñas y las controla el fabricante. Por otro lado, en la evaluación de produetos elaborados a partir del $100 \%$ de termoplásticos reciclados, de diferentes procedencias y sin haber sido sometido a un control apropiado de la conformidad de las materias primas, existe un bajo grado de confianza. Entre ambos extremos; el problema es mucho más complejo. El control del origen de los termoplásticos reciclados (desde el punto de vista de su conformidad a las especificaciones técnicas) y la verificación de la probable presencia de contaminantes o de cierta degradación toman, así, mayor importancia. El fabricante debe disponer de un sistema eficaz de verificación y control que le permita garantizar la obtención de un producto conforme.

\section{b) cantidad de materiales reciclados utilizados}

La cantidad de materiales reciclados utilizados en un producto regula el grado de confianza que acompaña a la evaluación de las prestaciones del producto. Pequeños porcentajes de termoplásticos reutilizados y reprocesados, mezclados con termoplásticos puros, pueden tener un efecto insignificante sobre las propiedades iniciales $y / 0$ futuras del producto. Pero el aumento del contenido de éstos conlleva una disminución de la confianza con el que pueden predecirse la vida útil y prestaciones del producto.

Las evaluaciones, $c$ n particular las relativas a la durabilidad, deben efectuarse sobre productos que contengan la cantidad más crítica de termoplásticos reciclados. Este grado crítico puede ser el porcentaje máximo previsto por el fabricante; pero pueden tomarse en consideración otros casos, con la condición de que estén acompañados de justificaciones apropiadas por parte del fabricante.

\section{c) historia térmica y de exposición de los materiales reciclados utilizados}

La mayoría de los termoplásticos utilizados en los productos presentados para su evaluación ofrecè una resistencia a la degradación muy limitada cuando no se estabilizan. Para combatir los efectos de la transformaeion y dotar al producto de una vida útil razonable, en las condiciones de uso previstas, los fabricantes adicionan distintos tipos de estabilizantes. La importante disminución del contenido en estabilizantes, durante la fabricación, utilización 0 transformación puede afectar mucho a las prestaciones, a largo plazo, del producto.

La evaluación de este efecto no puede bacerse por una simple medida de las propiedades inicialesdelosmaterintes en cuestión. Puede suceder que ligeras modificaciones, 
debidas a una exposición del material al calor o luz, imposibles de detectar a corto plazo, provoquen, cuando se prolonga la exposición, el nacimiento de puntos donde se inicie el proceso de degradación.

Por otro lado, un termoplástico reciclado que inicialmente contiene un alto porcentaje de plastificante, mayor del habitual, será útil para el producto en el que va a ser reutilizado. Si este contenido no se ha reducido ni ha sufrido degradación, el material en cuestión puede ofrecer una durabilidad mayor a la prevista. También es importante tener en cuenta la naturaleza, cantidad y compatibilidad de los estabilizantes presentes en los termoplásticos reciclados.

\section{d) uso final del producto}

El uso final previsto para el producto conteniendo termoplásticos reciclados constituye, igualmente, un criterio importante en la evaluación de su aptitud al uso. Hay que tener en cuenta la vida útil prevista, las consecuencias de las modificaciones en las prestaciones y el aspecto del producto, así como los agentes degradantes a los que existe riesgo de estar expuesto durante sư utilización. Puede admitirse un mayor contenido de termoplásticos reciclados para una aplicación que para otra.

Los perfiles de ventana de PVC rígido, p.ej., están expuestos durante su utilización y no se admiten cambios de color, debido a degradación superficial. Por ello, puede rechazarse la incorporación de importantes cantidades de termoplásticos reciclados en una extrusión uniforme. Por el contrario, un producto elaborado por coextrusión, con un alto contenido de termoplásticos reciclados en su núcleo y revestido de una envoltura de PVC rígido puro, podrá aceptarse, en función de la protección suministrada por la envoltura.

Las membranas de estanquidad están protegidas durante su uso, por lo que es probable que permanezcan relativamente estables durante su vida útil. En este tipo de productos, puede admitirse la presencia de una alto contenido en termoplásticos reciclados.

\subsection{Procedimientos de evaluación}

La evaluación, como si fueran puros, de productos conteniendo termoplásticos reciclados, no presenta dificultad suplementaria, ya que las propiedades esperadas de estos productos es idéntica a la de los productos fabricados a partir de termoplásticos puros. Se seguirán las Guías UEAtc existentes, en su caso. El principal problema radica en la evaluación de las prestaciones a largo plazo y la durabilidad. La ausencia de informaciones suficientes sobre procedencia, porcentaje e historia previa de los termoplásticos reciclados llevan a una disminución en el grado de confianza sobre las previsiones de las prestaciones a largo plazo del producto.
Se pedirá a los fabricantes aumentar los niveles de control sobre las materias primas recicladas. El origen de los materiales debe definirse (p. ej. seleccionado) y controlarse lo más posible. Así, será necesario efectuar verificaciones y controles sobre la naturaleza y propiedades de los termoplásticos en la recepción, sobre los métodos de reutilización adoptados, en su caso, sobre los plásticos reutilizados, reprocesados y el producto final. Los fabricantes deben disponer de un sistema de trazapilidad de los materiales reciclados a lo largo del ciclo de fabricación.

La evaluación de las prestaciones y la durabilidad debe efectuarse sobre el producto con el contenido más crítico de termoplástico reciclado previsto por el fabricante.

\subsection{Evaluación de la durabilidad}

El anexo A presenta un breve resumen de la acción de los agentes de envejecimiento sobre los termoplásticos reciclados con más frecuencia.

El anexo B propone una serie de características, cuya medida puede utilizarse para identificar los materiales o determinar los efectos debidos a la exposición y/o al reprocesado. Pueden ser apropiados otros métodos, en función de la naturaleza y /o uso final previsto.

Para establecer el efecto posible de la inclusión de termoplásticos reciclados sobre las propiedades a largo plazo de los productos, se emplean las técnicas tradicionales de envejecimiento. Implica la exposición de los productos a los agentes de envejecimiento (p.ej. calor, rayos UV, agua), solos o combinados, seguidos de la medida de propiedades significativas (p.ej. estabilidad de color, resistencia a la tracción, DHC, etc.). La elección del agente de envejecimiento y los parámetros a medir se basarán en la naturaleza y el uso final previsto.

La tabla 1 del anexo $\mathrm{C}$ indica el incremento del grado de incertidumbre creciente relativa a la naturaleza, calidad y adaptación de los termoplásticos reciclados en función de su origen. A medida que crece el grado de incertidumbre, el fabricante o sus suministradores deberán aplicar un control con el grado de exigencia correspondiente a las materias primas y al producto final.

La tabla 2 del anexo $\mathrm{C}$ indica la clasificación de uso final del producto en función de las solicitaciones a que se encontrará sometido durante su uso, seleccionando los métodos más apropiados del ensayo de durabilidad. Deben tenerse en cuenta, igualmente, las solicitaciones ligadas a las prestaciones.

Las informaciones suministradas por estas dos tablas no están basadas en estudios en profundidad, deben considerarse como guía. Cada caso debe evaluarse en función de sus características intrínsecas. 


\section{EVALUACIÓN BASADA EN LA PROCEDENCIA DE LOS TERMOPLÁSTICOS RECICLADOS}

\subsection{Termoplásticos reutilizados}

Se trata de la reutilización, pura y simple de un termoplástico en el mismo proceso de fabricación, por el fabricante inicial (p.ej. termoplásticos provenientes del reciclado primario) y así vuelve al mismo alimentador de la fabricación del material original. La cantidad de termoplásticos reutilizados incluida en un producto probablemente será baja, ya que una gran disponibilidad de termoplásticos reutilizados indicará que existen problemas en la producción. La única degradación que puede haber sufrido un termoplástico reutilizado es la fabricación inicial y la retransformación posterior. En cambio, si proviene de un rechazo, hay que tener en cuenta las razones, para evitar otros problemas sobre el producto final.

Los efectos de la fabricación inicial y la retransformación posterior pueden evaluarse midiendo las propiedades físicas y químicas significativas de los materiales reutilizados, comparándolas con las efectuadas sobre los materiales puros. Esto no será necesario más que una vez, cuando se prevé, por primera vez, el uso de los materiales reutilizados. Sin embargo, se realizarán ensayos de confirmación periódicamente.

En caso de modificaciones debidas a la reulilización del material, hay que verificar que el porcentaje de materiales reutilizados en el producto final no afecta al conjunto de sus propiedades, especialmente la prestación a largo plazo y la durabilidad. La evaluación de los productos que contienen termoplásticos reutilizados debe efectuarse sobre el producto final con el contenido más crítico previsto por el fabricante.

\subsection{Termoplásticos reprocesados}

El uso de termoplásticos reprocesados es análoga a la de los termoplásticos reutilizados. Sin embargo, ya que con los termoplásticos reprocesados el usuario no conoce bien el origen exacto y su naturaleza, se aumenta el riesgo de contaminación. Teniendo en cuenta, además, su mayor disponibilidad, los termoplásticos reprocesados pueden utilizarse, en mayor proporción, en los productos finales.

El fabricante necesita establecer una especificación técnica precisa que incluya las procedencias seleccionadas, características y tolerancias. Para asegurar la conformidad de los materiales reciclados a la especificación exigida deben efectuarse controles de calidad. Estos controles pueden realizarse a la recepción de los lotes o sobre la mezcla de material plástico puro/reprocesado antes de su conversión en producto final.

(c) Consejo Superior de Investigaciones Científicas

Licencia Creative Commons 3.0 España (by-nc)
En caso de reprocesado de residuos de su propio proceso, pero de productos con propiedades diferentes (p.ej. termoplásticos provenientes de reciclado secundario), la evaluación del fabricante debe ser la misma que para los termoplásticos reprocesados, a fin de asegurar la compatibilidad exigida.

La verificación de las propiedades del producto final deben efectuarse según los procedimientos rutinarios de control de calidad, pero no conviene olvidar la incidencia por la inclusión de materiales reprocesados sobre sus prestaciones a largo plazo y su durabilidad.

La evaluación, especialmente la de la durabilidad, debe efectuarse sobre el producto final con el contenido más crítico previsto por el fabricante.

\subsection{Termoplásticos reciclados después de su uso (origen industrial)}

Los termoplásticos de origen industrial reciclados después del uso pueden tener un único origen (botelleros de un fabricante, p.ej.) o varios, reagrupados por empresa especializada. Existe un factor adicional a considerar en estos materiales que ya han cumplido la función para la que fueron fabricados. El uso inicial debe considerarse cuando se evalúa su posible reciclado. Probablemente han estado expuestos a la acción de agentes de degradación y sufrido una alteración de sus propiedades físicas y/o químicas. Igualmente pueden haber estado en contacto con agentes contamínantes (productos alimenticios, químicos, etc.) capaces de afectar gravemente su prestación una vez reprocesados. También existe el riesgo de que sean mezclados con otros tipos de termoplásticos.

Los niveles de control exigidos para estos materiales son más estrictos que para los termoplásticos reutilizados reprocesados. Se necesita incluir procedimientos apropiados de selección y tratamiento para excluir cualquier contaminante. También deben permitir comprobar la ausencia de otros termoplásticos, salvo que se haya demostrado que la inclusión de éstos no tenga influencia negativa sobre la prestación del producto final. En este caso, el fabricante debe indicar en sus especificaciones, la naturaleza y porcentaje de los termoplásticos considerados como aceptables.

Este procedimiento de selección y tratamiento puede ser dificil de controlar sin la existencia de un sistema de control de calidad complejo y laborioso. La garantía de una conformidad continua a las especificaciones exigirá controles muy frecuentes.

La evaluación, especialmente la de durabilidad, debe efectuarse sobre el producto final con el contenido más crítico previsto por el fabricante. 
Si los termoplásticos reciclados provienen de distintos orígenes seleccionados, el fabricante debe instalar en su empresa un sistema de separación que evite la entrada en producción de materiales que no hayan pasado dichos controles.

\subsection{Termoplásticos reciclados después de su uso (origen doméstico)}

El uso de termoplásticos reciclados de origen doméstico reciclados después de su uso es análoga a la de los materia- les que provienen de origen industrial (ver 5.3). Ya que las basuras domésticas están menos controladas (p.ej. puntos de recogida de residuos urbanos), existe mayor riesgo de la presencia de contaminantes y otros termoplásticos. Salvo casos específicos, hacen bastante costosa su utilización, por las dificultades de selección, limpieza y control.

La evaluación de este tipo de producto debe efectuarse con especial cuidado. Los niveles de control a adoptar por el fabricante o sus suministradores deben ser los más estrictos de todas las categorías (Cuadro 1).

\section{CUADRO 1}

Origen de los termoplásticos reciclados

\begin{tabular}{|c|c|c|c|c||}
\hline \multicolumn{2}{|c|}{ Termoplásticos reciclados antes de su uso } & \multicolumn{2}{c|}{ Termoplásticos reciclados después de su uso } \\
\hline \multicolumn{2}{|c|}{ Termoplásticos reutilizados } & $\begin{array}{c}\text { Termoplásticos } \\
\text { reprocesados }\end{array}$ & Origen industrial & Origen doméstico \\
\hline $\begin{array}{c}\text { reciclado } \\
\text { primario }\end{array}$ & $\begin{array}{c}\text { reciclado } \\
\text { secundario } \\
\text { reciclado } \\
\text { primario o } \\
\text { secundario }\end{array}$ & $\begin{array}{c}\text { reciclado primario } \\
\text { o secundario }\end{array}$ & $\begin{array}{c}\text { reciclado primario } \\
\text { o secundario }\end{array}$ \\
$\begin{array}{l}\text { Material del producto } \\
\text { original (p.ej. colas, } \\
\text { orillos, rebabas, etc.) }\end{array}$ & $\begin{array}{l}\text { residuos de su propio } \\
\text { proceso, pero de } \\
\text { productos con } \\
\text { propiedades diferentes }\end{array}$ & $\begin{array}{l}\text { materiales no usados, } \\
\text { recogidos } y \\
\text { suministrados } \\
\text { por un tercero }\end{array}$ & $\begin{array}{l}\text { materiales de productos } \\
\text { usados de ori gen } \\
\text { industrial (p.ej. } \\
\text { embalajes, tapones, } \\
\text { residuos de } \\
\text { demoliciones) }\end{array}$ & $\begin{array}{l}\text { materiales de productos } \\
\text { usados provenientes del } \\
\text { sistema de recogida de } \\
\text { basuras (p.eje. botellas } \\
\text { de PVC) }\end{array}$ \\
\hline Ver 5.1
\end{tabular}

\section{ANEXOS}

\section{A) Efecto de los agentes de envejecimiento sobre los termoplásticos}

Los agentes de envejecimiento tienen efectos específicos sobre los termoplásticos. El presente anexo ofrece un breve resumen de sus posibles efectos sobre los termoplásticos mas comúnmente reciclados:

\section{Polietileno y polipropileno}

\subsection{Oxidación térmica}

En ausencia de oxígeno el polietileno y polipropileno permanecen estables hasta que alcanzan altas temperaturas. Pero la presencia de oxígeno conlleva a una oxidación térmica (degradación de radicales libres) que provocan endurecimiento, oscurecimiento y disminución de peso molecular. Proceso relativamente lento a baja temperatura, pero que se acelera con el aumento de la temperatura. La reducción del peso molecular disminuye con la serie $\mathrm{PP}>\mathrm{LDPE}>$ copolímero $\mathrm{PE} / \mathrm{PP}>\mathrm{HDPE}$, en función de un aumento en la relación entre las reacciones de reticulación y rotura de cadena.

\section{$1.2 \mathrm{Luz}$}

En presencia de oxígeno, los rayos UV provocan, en las poliolefinas, los mismos efectos de degradación que el calor, pero con mayor velocidad. La iniciación de estas reacciones de degradación se producen a temperaturas relativamente bajas. La foto-oxidación de las poliolefinas es, con mucho, el factor más crítico en la degradación de las propiedades. 
Los efectos de degradación de las poliolefinas, debidas a los rayos UV, pueden combatirse mediante la utilización de estabilizantes y/o negro de humo.

\subsection{Agua}

Estos dos termoplásticos son relativamente resistentes a los efectos del agua. Los LDPE, en ausencia de solicitaciones mecánicas, pueden resistir el agua caliente, mientras que el HDPE y PP, en ausencia de cualquier solicitación, pueden resistir el agua hirviendo.

\subsection{Productos químicos}

Tanto el PE como el PP son resistentes a los efectos de los ácidos no oxidantes, álcalis y la mayoría de las soluciones acuosas. El ácido nítrico concentrado oxida el material, conduciéndole a una disminución de las propiedades mecánicas y a un incremento del factor de potencia.

Pueden producirse agrietamientos en algunas calidades de estos termoplásticos, cuando se someten a tensiones mecánicas estando en contacto con algunos productos químicos, tales como: alcohol, hidrocarburos líquidos, ésteres orgánicos, jabones metálicos, aceites de silicona, etc.

\section{Policloruro de vinilo (PVC rígído y plastificado)}

\subsection{Calor}

La degradación térmica del PVC es un proceso complejo e implica la deshidrocloración (DHC) de las cadenas poliméricas, así como reacciones de reticulación y rotura de cadena. Pero el efecto del calor sobre el PVC puede controlarse mediante la adición de estabilizantes apropiados.

La degradación del PVC se manifiesta por una disminución de las propiedades mecánicas y cambio de color. Éste constituye el efecto más evidente, el blanco vira a amarillo pálido, naranja, marrón, hasta llegar a negro. La deshidrocloración se produce en los primeros pasos de la degradación del material, la medida del grado de DHC puede utilizarse para evaluar la aptitud al uso del termoplástico reciclado considerado.

El calor también puede afectar a los PVC plastificados, acelerando la migración y pérdida de plastificantes, lo que lleva a un endurecimiento del producto.

\section{$1.2 \mathrm{Luz}$}

El efecto de los rayos UV sobre los PVC es análogo al del calor, comprende los mismos procesos de degradación. Se trata de la forma de degradación más crítica para los productos de construcción usados en el exterior.

\subsection{Agua y productos químicos}

Los PVC generalmente presentan una excelente resistencia al agua y productos químicos. Sin embargo, conviene asegurar que los aditivos utilizados en los compound sean igualmente resistentes a estos agentes, para evitar que dichos aditivos no se eliminen por lixiviación.

\section{B) Características para la identificación o evaluación}

Se proponen las siguientes características para la identificación de los materiales o la determinación de los efectos de exposición y/o reprocesado (lista no completa)

\section{Polietileno y polipropileno}

- Nivel de oxidación (materia prima), mediante:

- temperatura de endurecimiento (PP, materia prima y producto final)

- espectroscopía IR transformada de Fourier *

- Densidad (materia prima)

- Punto de reblandecimiento (materia prima)

- Contenido en negro de humo (en su caso, materia prima y producto final)

- Contenido en cenizas (presencia de una carga, producto final)

\section{Policloruro de vinilo (rígido)}

- Contenido en PVC

. Densidad (materia prima)

- Grado Vicat (producto final)

- Tamaño de partículas (impurezas y origen de los estabilizantes, materia prima)

. Índice $\mathrm{K}^{*}$ (materia prima)

- Espectroscopía IR transformada de Fourier * (materia prima)

- Resistencia a la deshidrocloración (DHC) (producto final)

. Cambio de color por exposición a los UV (producto final)

\section{Policloruro de vinilo (plastificado)}

(teniendo en cuenta un buen conocimiento del origen del material)

- Plastificantes - naturaleza y contenido (materia prima) 
- Densidad (materia prima),

- Estabilidad del color (retención de estabilizantes)

- Resistencia a la deshidrocloración (DHC) (producto final)

- Contenido en plastificantes (producto final)

. Espectroscopía IR transformada de Fourier*

\section{C) Recomendaciones relativas al grado de control de calidad y a la importancia de la acción de los agentes ambientales}

La tabla 1 recoge los niveles de incertidumbre crecientes (A a D) debido a la naturaleza y calidad de los termoplásticos reciclados en función de su origen, así como los niveles de control exigidos. En todos los casos, será necesario definir una especificación técnica precisa, que servirá como referencia, tanto de las materias primas como del producto final. En último caso, se referirá a las Guías de la UEAtc existentes.
Leyenda:

A = Material proveniente del mismo producto de partida, que no necesita de verificaciones o'controles suplementarios.

B = Grado normal de control de calidad para materias primas (certificado de conformidad, ensayos sencillos, etc.).

$\mathrm{C}=$ Grado especial de control de calidad a la recepción de las materias primas; detección de contaminantes y otros plásticos inaceptables; control de conformidad a las especificaciones de las materias primas o mezclas. Puede pedirse una verificación de la prestación a largo plazo del producto final.

$\mathrm{D}=$ Como el $\mathrm{C}$, pero con controles/verificaciones más frecuentes. La periodicidad de estos controles puede estar en función del proceso de selección adoptado, pero sobre cada lote.

TABLA 1

Nivel de control de calidad en función del origen de los termoplásticos reciclados

\begin{tabular}{||l|l|c||}
\hline Termoplásticos reciclados & Origén & Nivel de control de calidad \\
\hline $\begin{array}{l}\text { Termoplásticos reciclados } \\
\text { antes de su uso }\end{array}$ & Termoplásticos reutilizados & A \\
\cline { 2 - 3 } $\begin{array}{l}\text { Termoplásticos reciclados } \\
\text { después de su uso }\end{array}$ & Termoplásticos reprocesados & B \\
\hline & $\begin{array}{l}\text { De origen industrial y fuente } \\
\text { única }\end{array}$ & C \\
\cline { 2 - 3 } & $\begin{array}{l}\text { De origen industrial y varias } \\
\text { fuentes (pero seleccionadas) }\end{array}$ & D \\
\hline
\end{tabular}

1 Los termoplásticos reciclados después de uso de origen doméstico, en circunstancias especiales y con selección y limpieza apropiadas, pueden evaluarse de la misma forma que los provenientes de origen industrial.

2 El uso de termoplásticos reciclados de origen seleccionado están excluidos en este documento. 
La tabla 2 recoge los niveles de solicitaciones crecientes ( 1 a 5$)$ a los que puede ser sometido el producto final en su ambiente de uso. Las solicitaciones relacionadas con la función del producto deben ser tenidas en cuenta (p.ej. las canalizaciones pueden estar sometidas a presión).

\section{D) Ejemplos de productos de construcción susceptibles de incluir termoplásticos reciclados}

\section{Polietileno}

- Canalizaciones de evacuación enterradas

- Membranas de estanquidad y bandas hidrófobas

- Placas bajo cubierta

- Barreras de vapor

- Perfiles extruidos

\section{Polipropileno}

. Canalizaciones de evacuación enterradas
- Membranas de estanquidad y bandas hidrófobas

- Placas bajo cubierta

- Barreras de vapor

- Perfiles extruidos

\section{Policloruro de vinilo (rígido)}

- Canalizaciones de evacuación enterradas

- Perfiles extruidos (ventanas, goterones, bandejas, subcapas, etc.)

- Tuberías y productos de drenaje

- Productos de moldeo

- Conducciones y canalizaciones eléctricas

\section{Policloruro de vinilo (plastificado)}

- Revestimientos de suelos

- Membranas de estanquidad

${ }^{(*)}$ Ensayo dificil de realizar y que necesita de un equipo del que puede no dispongan algunos fabricantes y organismos de evaluación.

TABLA 2

Solicitaciones relacionadas con el ambiente

\begin{tabular}{|c|c|c|l||}
\hline Código & Nivel UV & Nivel de temperatura & \multicolumn{1}{c|}{ Solicitaciones (ejemplos) } \\
\hline 1 & Alta & Alta & $\begin{array}{l}\text { Productos de color oscuro expuestos al } \\
\text { exterior }\end{array}$ \\
\hline 2 & Alta & Moderada & $\begin{array}{l}\text { Productos de color claro expuestos al } \\
\text { exterior }\end{array}$ \\
\hline 3 & Ninguna & Moderada/Débil & $\begin{array}{l}\text { Productos enterrados (p.ej. tubería de } \\
\text { agua caliente) }\end{array}$ \\
\hline 4 & Débil & Moderada/Débil & $\begin{array}{l}\text { Productos interiores (p.ej. perfiles } \\
\text { interiores }\end{array}$ \\
\hline 5 & Ninguna & Moderada/Débil & $\begin{array}{l}\text { Productos enterrados (p.ej. membranas } \\
\text { de estanquidad) }\end{array}$ \\
\hline
\end{tabular}

